\title{
A Novel Technique of ECG Denoising based on Lifting Wavelet Transform and Total Variation Minimization
}

Talbi Mourad ( $\square$ mouradtalbi196@yahoo.fr)

Centre de Recherches et Technologies de l'Energie

Baazaoui Riadh

Universite de Tunis El Manar

\section{Research}

Keywords: Lifting Wavelet Transform, ECG, Total Variation Minimization, Thresholding

Posted Date: June 3rd, 2020

DOI: https://doi.org/10.21203/rs.3.rs-30285/v1

License: (c) (i) This work is licensed under a Creative Commons Attribution 4.0 International License.

Read Full License 


\title{
A Novel Technique of ECG Denoising based on Lifting Wavelet Transform and Total Variation Minimization

\author{
Mourad Talbi ${ }^{1}$, Riadh Baazaoui ${ }^{2}$ \\ ${ }^{1}$ Laboratoire des Semi-Conducteurs, Nanostructures et Technologie Avancée \\ ${ }^{1}$ Center of Researches and Technologies of Energy of Borj Cedria, Tunis 952050, Tunisia \\ ${ }^{2}$ Faculty of Sciences of Tunis, University Tunis El-Manar Tunis, 1060, Tunisia \\ talbi1969@yahoo.fr
}

\begin{abstract}
:
- Buckground:

The signal of Electrocardiogram $(E C G)$ is one of the most popular diagnostic means providing an electrical picture of the heart and also information about different pathological conditions. Due to the path deformities and external electrical disturbances, the signal of ECG becomes noisy. Hence, in literature, many ECG denoising algorithms have been proposed and among them we can mention the techniques based on wavelet coefficient shrinkage. The purpose of this paper is to denoise ECG signals applying a new ECG Denoising technique and proving its performance compared to some denoising approaches existing in literature. This new proposed technique of ECG Denoising consists at the first step in applying the Lifting Wavelet Transform ( $L W T$ ) to the noisy ECG Signal (where 2 is the decomposition level) in order to obtain three noisy wavelet sub-bands, $c D_{1}, c D_{2}$ and $c A_{2}$. The two coefficients, $c D_{1}$, $c D_{2}$ are details ones and they are denoised by soft or hard thresholding in order to obtain denoised coefficients, $c D d_{1}$ and $c D d_{2}$. The coefficient $c A_{2}$ is an approximation one and is denoised by Total Variation Minimization (TVM) in order to obtain a denoised one, $c A d_{2}$. Finally, the inverse of $L W T$ is applied to $c D d_{1}, c D d_{2}$ and $c A d_{2}$ in order to obtain the denoised ECG signal. The evaluation of this proposed technique is performed by comparing it to three other denoising approaches existing in literature. The first one of these approaches is based on TVM, the second one is $1-D$ double-density complex DWT denoising method and the third one is based on non local means.
\end{abstract}

\section{- Results:}

All These techniques are applied on a number of ECG signals taken from $M I T-B I H$ database and corrupted by an additive White Gaussian noise at different values of Signal to Noise Ratio ( $S N R$ ). The obtained results from the computation of the $S N R$ and the Mean Square Error $(M S E)$, show that the proposed technique outperforms the other three mentioned techniques.

\section{- Conclusion}

In this paper, the proposed ECG denoising technique based on $L W T$ and TVM, outperforms the other previously mentioned denoising approaches and this based on the computation of the SNR and MSE. 
Keywords: Lifting Wavelet Transform, ECG, Total Variation Minimization, Thresholding.

\section{Buckground}

The signal of ECG (Electrocardiogram) is one of the most popular diagnostic means providing an electrical picture of the heart and also information about different pathological conditions. Those signals originate from the heart and pass through the tissues with diverse characteristics and reach up to the several recording leads placed on the subject skin [1]. Due to the path deformities and external electrical disturbances, the signal of $E C G$ becomes noisy [1]. The typical noises in the ECG signal include muscle noise, power-line interference, baseline wander, and etc. Nowadays, the biotelemetry has become a dominant means of monitoring the cardiac condition of ambulatory patients [2,3]. Also, for detecting arrhythmias and cardiac abnormalities, wireless ambulatory ECG recording is actually routinely employed [4]. In such cases, the ECG data is sent through the channel (telephone lines, wireless and etc.) to a remote location where it is analysed [1]. In this process, it gets corrupted by the noise caused by channel. For the correct diagnosis, the noise cancellation from such $E C G$ signals is required.

In literature, have been proposed many algorithms for $E C G$ signal denoising $[5,6,7,8,9,10]$. Among those algorithms we can mention the techniques based on discrete wavelet coefficient shrinkage [6, 10, 11]. Also the techniques based on the Empirical Mode Decomposition $(E M D)[5,9]$. Concerning the techniques based on Discrete Wavelet coefficients Shrinkage, we obtain a good estimate of the clean ECG signal by eliminating the lower magnitude $D W T$ coefficients and then applying the inverse of DWT [1]. Concerning the techniques based on EMD, the estimate of the clean ECG signal is obtained by eliminating first few intrinsic mode functions (IMFs) since these account for the high frequency variations present in the signal [1]. Although, this process is reported to distorts the $Q R S$ complexes [1]. In [12] the portions of the first few IMFs those correspond to the QRS complexes are preserved by means of a Tukey window. In [13] a hybrid EMD-wavelet approach combining the windowed EMD with wavelet soft-thresholding was proposed to further ameliorate the denoising performance.

The NLM (Nonlocal Means) technique [14] is a very effective image denoising approach. Actually, it is applied for ECG signal denoising [15] and it is shown that it outperforms the hybrid $E M D$-wavelet approach and this for a number of ECG signals. The NLM technique was originally developed for image denoising with the supposition that the underlying clean image owns several pixels with similar neighborhood. In normal cases, the $E C G$ signals are almost structurally repetitive and therefore owns such redundancy. In one-dimensional NLM denoising technique proposed in [15], the estimates of the underlying clean signal samples are obtained by weighted averaging of the samples having similar neighborhoods. The applied weighting is proportional to the similarity in the neighborhood and is independent of the temporal location of the samples. As a result, the samples with quite similar neighborhoods are given higher weights however lower weights are assigned to the samples with dissimilar neighborhoods [1]. Consequently, it exploits directly the nonlocal similarity existing in the signal. The NLM algorithm employs a sample-based approach in which each sample is independently estimated. In other terms, a sample estimate at one location doesn't contribute to the estimation of other samples even if those are in close proximity. The nonlinear filtering techniques such as the shrinkage of the discrete wavelet coefficients do not face such disadvantage as these rely on the inherent sparsity of the clean signal in the transform domain. Though, the DWT shrinkage based approaches couldn't exploit the nonlocal redundancy existing in the signal. On combining the transform based approach and the block-based NLM technique, their relative advantages can be exploited. The alike idea has been already 
explored for image denoising [16, 17, 18, 19]. However, is yet to be explored for the biomedical signals like ECG,EEG and etc. In [1] was we proposed an ECG denoising technique which exploits local as well as nonlocal similarity in the signal. In [1], the similar blocks of samples are estimated in a collaborative manner. The denoising is performed by the shrinkage of the two-dimensional $(2-D) D W T$ coefficients of the matrix formed with these similar blocks [1]. This process is repeated for each of the overlapping blocks providing several estimates for a sample. The final estimate is obtained by averaging those estimates [1]. In this paper we propose a new ECG denoising technique based on Lifting Wavelet Transform (LWT) [20, 21, 22] and TVM [23]. The rest of this paper is organized as follow: in section 2, we will deal with the Lifting Wavelet Transform $(L W T)$. In section 3, we will deal with the Total Variation Minimization, in section 4 we will detail the proposed technique. In section 5 we will deal with the $1-D$ double-density complex $D W T$ denoising method [25]. In section 6, we will detail the Denoising approach Based on Nonlocal Means [26, 27]. In section 7, we will present results and discussion. Finally in section 8 , we will conclude.

\section{The Lifting Wavelet Transform (LWT)}

The $L W T$ was introduced by Swelden and becomes a powerful tool for signal and image analysis. In fact, the $L W T$ has a faster and efficient implementation compared to the Discrete Wavelet Transform (DWT). Also compared to DWT, the $L W T$ leads to better results in the image denoising domain and also in image compression and watermarking domains. The LWT saves times and has a better frequency localization feature that overcomes the shortcomings of DWT. The Signal decomposition by $L W T$ necessitates three steps which are splitting, prediction and update and are detailed as follow:

- The signal splitting is to divide the original signal $x(n)$ into non overlapping odd and even samples which are respectively $x_{0}(n)$ (odd samples) and $x_{e}(n)$ (even samples):

$$
\left\{\begin{array}{c}
x_{e}(n)=x(2 n) \\
x_{o}(n)=x(2 n+1)
\end{array}\right.
$$

- The Prediction is summarized as follow:

If both even and odd samples are correlated, then one can be the predictor of the other. In the prediction of even sample $\left(x_{o}(n)\right)$, the odd sample $\left(x_{e}(n)\right)$ is used as follow:

$$
d(n)=x_{o}(n)-P\left(x_{e}(n)\right)
$$

With the difference $d(n)$ between the original sample and the predicted value, is defined as a high frequency component and $P(\cdot)$ is the predictor operator.

- The updating: with the help of the detail signal $d(n)$ and the update operator $(U(\cdot))$, one can update the even samples. The low frequency components $l(n)$ are then representing the coarse shape of the original signal. They are formulated as follow:

$$
l(n)=x_{e}(n)+U(d(n))
$$

Those steps are illustrated in Figure 1. 


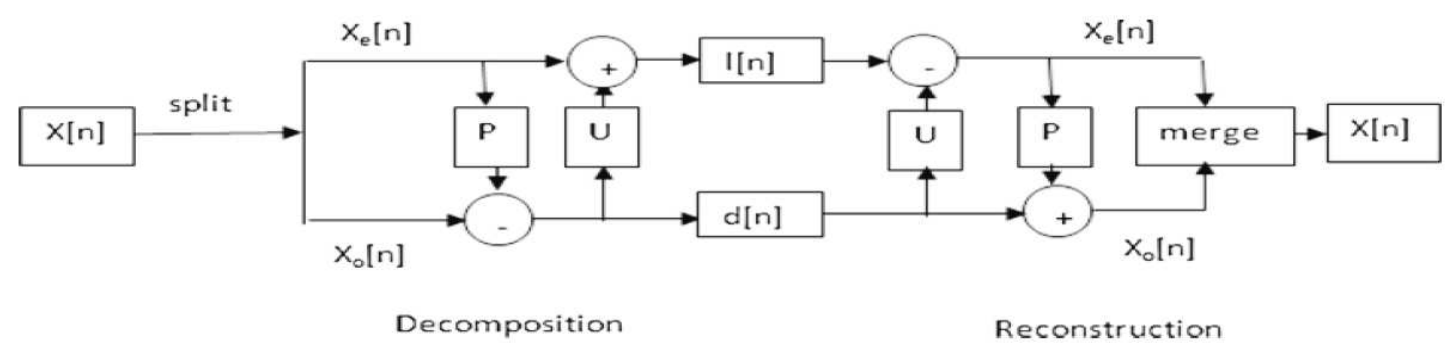

Figure 1. Decomposition and Reconstruction of a signal X[n] using $L W T$ and its inverse respectively.

\section{The Total Variation Minimization}

The Total Variation Minimization consists to solve the lagrangian TV minimization (RudinOsher-Fatemi) [24]:

$x t v=\operatorname{argmin}_{u}\left(\frac{1}{2} \cdot\|x-u\|^{2}+\lambda \cdot T V(u)\right)$

Where $x t v$ is the denoised signal and $T V$ is the discrete $T V$ norm $1-D: T V(u)=$ $\sum_{i}^{N-1}|u(i)-u(i-1)|$.

\section{The proposed $E C G$ Denoising Technique}

As previously mentioned, in this paper we propose a new technique of ECG Denoising based on Lifting Wavelet Transform (LWT) [20, 21, 22] and Total Variation Minimization (TVM) [23]. This technique consist at first step in applying the $L W T$ to the noisy ECG Signal (where 2 is the decomposition level) in order to obtain three wavelet sub-bands, $c D_{1}, c D_{2}$ and $c A_{2}$. The two coefficients, $c D_{1}, c D_{2}$ are details coefficients and they are denoised by soft or hard thresholding. The coefficient $c A_{2}$ is an approximation coefficient and is denoised by TVM [23]. In Figure 2 is illustrated the flowchart of the proposed technique.

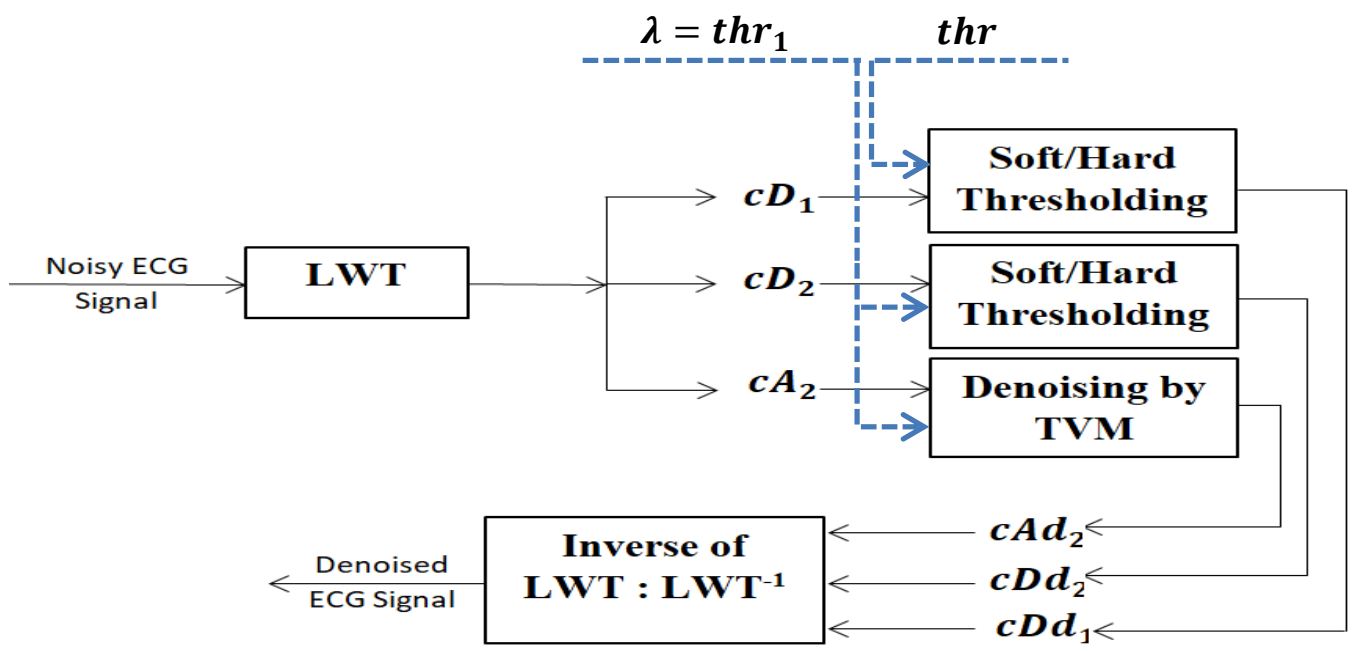

Figure 2. The flowchart of the proposed ECG Denoising Technique.

According to this figure, we first apply the $L W T$ to the noisy $E C G$ signal in order to obtain two noisy details coefficients, $c D_{1}$ and $c D_{2}$ and one noisy approximation coefficient, $c A_{2}$. Then $c D_{1}$ and $c D_{2}$ are thresholded using Soft/Hard Thresholding in order to obtain two denoised $c D d_{1}$ and $c D d_{2}$. Also the approximation coefficient, $c A_{2}$ at level 2 , is denoised by 
TVM technique [23] in order to obtain the denoised approximation coefficient, $c A d_{2}$. Finally, the denoised ECG signal is obtained by applying the inverse of $L W T, L W T^{-1}$ to the denoised coefficients, $c D d_{1}, c D d_{2}$ and $c A d_{2}$.

According to this figure, the thresholding of the noisy detail coefficient $\left(c D_{1}\right)$ requires the estimation of noise and then computing the threshold, $t h r$. In this work we use for computing this threshold, thr, the following equation:

$\operatorname{thr}=\sigma \times \sqrt{2 \times \log (N)}$

Where $N$ is the samples number in the details coefficient at the first level, $c D_{1}$. The parameter $\sigma$ is the noise level and is estimated using the following equation:

$\sigma=M A D\left(\left|c D_{1}\right|\right) / 0.6745$

Also according to this figure, the thresholding of the noisy detail coefficient $\left(c D_{2}\right)$ requires the estimation of noise and then computing the threshold, $t h r_{1}$. In this work we use for computing this threshold, $t h r_{1}$, the following equation:

$t h r_{1}=\sigma_{1} \times \sqrt{2 \times \log \left(N_{1}\right)}$

Where $N_{1}$ is the samples number in the details coefficient at the second level, $c D_{2}$. The parameter $\sigma_{1}$ is the noise level and is estimated using the following equation:

$\sigma_{1}=M A D\left(\left|c D_{2}\right|\right) / 0.6745$

Also according to this figure, the application of the Denoising Technique TVM [23] to the noisy approximation coefficient $\left(c A_{2}\right)$, requires a parameter, $\lambda$ which controls how much denoising you want. In this work, this parameter is chosen to be equal to $t h r_{1}$ (Figure 2 and eq. (6)).

In section 7, is made a comparative study between the proposed $E C G$ denoising technique, the denoising approach based on TVM [23, 24], the $1-D$ double-density complex DWT denoising method [25] and the ECG denoising technique based on non local means [26, 27]. The $1-D$ double-density complex DWT denoising method [25] is detailed in section 5. The second denoising technique based non local means $[26,27]$ is detailed in section 6 .

\section{The $1-D$ double-density complex $D W T$ denoising method [25]}

The input signal $x(n)$ is processed by two parallel iterated filter banks $h_{i}(n)$ and $g_{i}(n)$ with $i=0,1,2$. The real part of a complex wavelet transform is produced by the subband signals of the upper $D W T$ and the imaginary part is produced by the lower $D W T$ as in Figure 3. The implementation process for the $1-D$ double density complex $D W T$ is illustrated as a flowchart in Figure 3. 


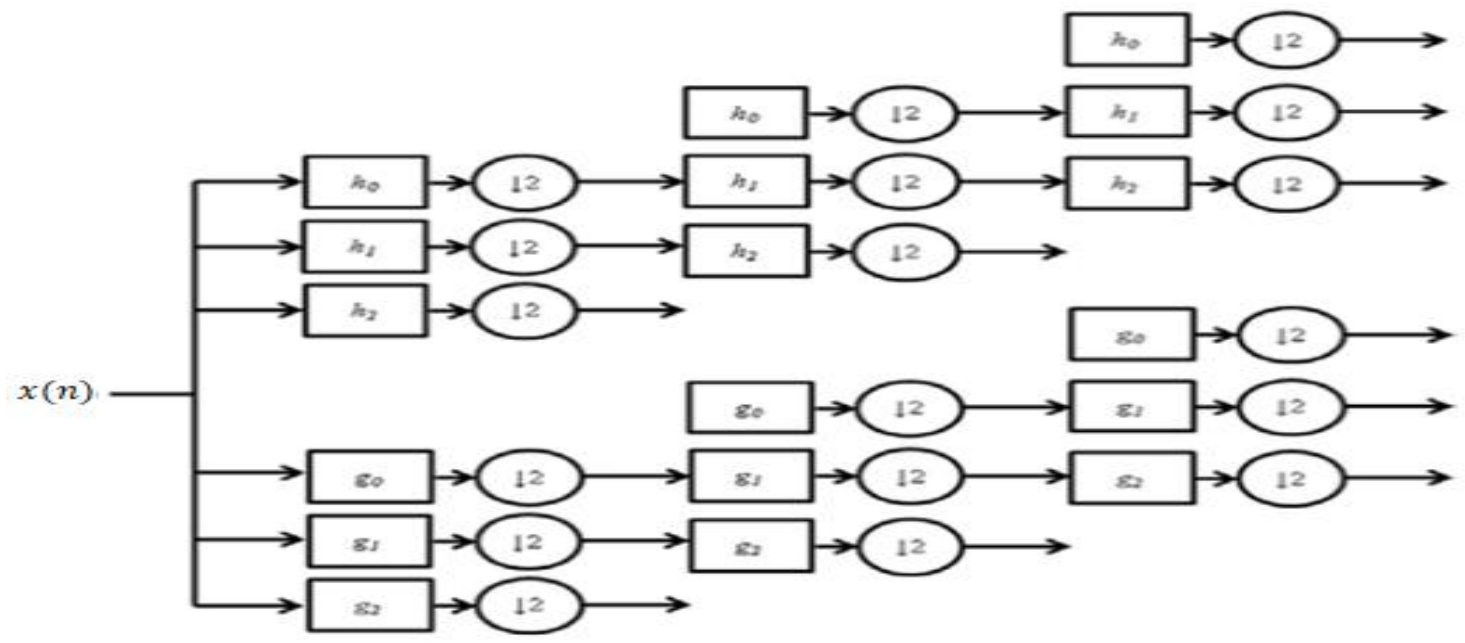

Figure 3. Filter bank diagram of $1-D$ double density complex $D W T$.

The $1-D$ double-density complex $D W T$ denoising technique can be summarized by the flowchart illustrated in Figure 4.

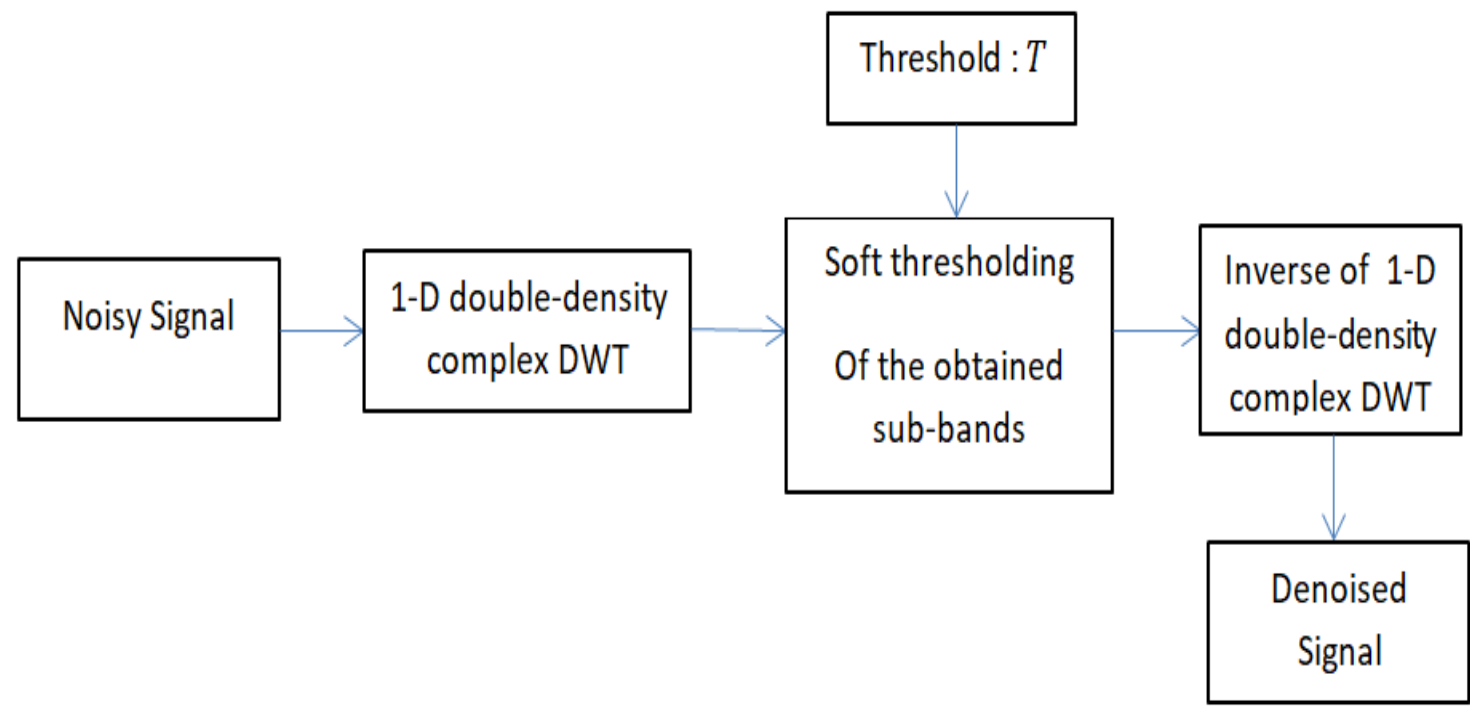

Figure 4. Signal denoising using $1-D$ double-density complex $D W T$ denoising technique.

As shown in this figure, the different steps of this denoising technique are listed as follow:

- First step: Apply the $1-D$ double-density complex $D W T$ to the noisy,

- Second step: Apply the soft thresholding to the subbands obtained in the first step. The soft thresholding uses a threshold, $T$,

- Third Step: Apply the inverse of $1-D$ double-density complex DWT to the denoised sub-bands obtained in the second step and this in order to obtain the denoised signal.

\section{The Denoising approach Based on Nonlocal Means [26, 27]}


In their work [26, 27], Brian H. Tracey and Eric L. Miller have applied the non local means for ECG denoising. Non Local means permits to address the problem of recovering the clean signal, $s$ from the noisy signal, $x$ :

$x=s+n$

Where $n$ is an additive noise. For a given sample $k$, the estimate $\hat{s}(k)$ is a weighted sum of the values at others samples $j$ which are belonging to some search neighbourhood $N(k)$ [26, 27].

$\hat{s}(k)=\frac{1}{Z(k)} \sum_{j \in N(k)} w(k, j) v(j)$

Where $Z(k)=\sum_{j} w(k, j)$ and the weights are expressed as follow [26, 27]:

$w(k, j)=\exp \left(-\frac{\sum_{\delta \in \Delta}(x(k+\delta)-x(j+\delta))^{2}}{2 L_{\Delta} \lambda^{2}}\right)$

$w(k, j)=\exp \left(-\frac{d^{2}(k, j)}{2 L_{\Delta} \lambda^{2}}\right)$

Where $\lambda$ designates a bandwidth parameter and $\Delta$ is a local patch of samples surrounding $k$, including $L_{\Delta}$ samples; a patch having the same shape also surrounds $j$. In [26, 27], $d^{2}$ denotes the summed, squared point-by-point difference between samples in the patches cantered on the samples $k$ and $j$. In [26, 27], each patch is averaged with itself with weight $w(k, k)=1$. To achieve a smoother result, a canter patch correction is frequently applied [26, 27]:

$w(k, k)=\max _{j \in N(k), j \neq k} w(k, j)$

The denoising approach based on nonlocal means [26, 27] requires the estimation of noise level, $\sigma_{1}$. Therefore, in this work we have applied the discrete wavelet transform (DWT) to the noisy ECG signal in order to estimate $\sigma_{1}$ using the following equation:

$\sigma_{1}=M A D(|c D 1|) / 0.6745$

Where $c D 1$ represents the details coefficient obtained from the application of the $D W T$ to the noisy ECG signal.

The value of $\sigma_{1}$ is then multiplied by 0.6 and the obtained value is used in the application of the denoising approach based on nonlocal means.

\section{Results and discussion}

As previously mentioned, a comparative study is performed between the proposed technique with Soft/Hard thresholding and others denoising ones which are the denoising approach based on TVM [23, 24], the 1-D double-density complex DWT denoising method [25] and the ECG denoising technique based on non local means $[26,27]$. This study is made by the computation of Signal to Noise Ratio $(S N R)$ and Mean Square Error (MSE). These different denoising techniques are applied on seven noisy ECG signals. Those signals are in number of 35 and each of them is obtained by artificially corrupting one of cleans ECG signals by an additive Gaussian Noise with some value of SNR before denoising (SNRi). This value can be 
chosen from -5 to $15 d B$ with a step of $5 d B$. The cleans ECG signals are taken from MIT - BIH database and are seven signals: 105.dat, 107.dat, 109.dat, 123.dat, 124. dat, 200. dat and 201.dat. In Tables 1 and 2, are listed the results obtained from the computation of the $S N R$ after denoising, SNRf and the MSE between the clean and the denoised ECG signals. Those results are the mean values where each of them is computed from seven values of $S N R f / M S E$ and this for each value of $S N R i$.

Table 1. Comparative study in term of Signal to Noise Ratio (SNR): results obtained from the computations of the mean of seven values of SNRf (SNR after denoising). This mean is computed for seven clean ECG signals $(105,107,109,123,124,200$ and 201).dat corrupted by Gaussian white noise with different values of $S N R i$ before denoising (varying from -5 to $15 d B$ with step of $5 d B$ ).

\begin{tabular}{|c|c|c|c|c|c|}
\hline The Denoising & \multicolumn{5}{|c|}{ SNRi (dB) } \\
\cline { 2 - 6 } Technique & -5 & 0 & 5 & 10 & 15 \\
\hline $\begin{array}{c}\text { SNRf obtained by } \\
\text { the proposed ECG } \\
\begin{array}{c}\text { Denoising } \\
\text { technique (Hard } \\
\text { thresholding) }\end{array}\end{array}$ & 5.2435 & 9.2255 & 13.6082 & 17.4077 & 20.6016 \\
\hline $\begin{array}{c}\text { SNRf obtained by } \\
\text { the proposed ECG } \\
\begin{array}{c}\text { Denoising } \\
\text { technique (Soft } \\
\text { thresholding) }\end{array}\end{array}$ & $\mathbf{5 . 6 3 5 9}$ & $\mathbf{9 . 8 9 6 3}$ & $\mathbf{1 4 . 0 2 5 5}$ & $\mathbf{1 7 . 6 4 9 8}$ & 20.6448 \\
\hline $\begin{array}{c}\text { SNRf obtained by } \\
\text { the TVM } \\
\text { technique [23, 24] }\end{array}$ & 2.4137 & $\mathbf{6 . 4 3 9 1}$ & 10.9663 & 15.6451 & 20.3097 \\
\hline $\begin{array}{c}\text { SNRf obtained by } \\
\text { 1D double-density } \\
\text { complex DWT } \\
\text { denoising method } \\
\text { [25] }\end{array}$ & $\mathbf{4 . 4 7 0 3}$ & 8.7680 & 13.1836 & 17.2554 & 21.0807 \\
\hline $\begin{array}{c}\text { SNRf obtained by } \\
\text { the ECG denoising } \\
\text { technique based on } \\
\text { non local means } \\
\text { [26, 27] }\end{array}$ & 4.8513 & 9.1454 & 13.0091 & 16.9997 & $\mathbf{2 1 . 1 5 5 0}$ \\
\hline
\end{tabular}

According to this table, the values in red color are the highest values of $S N R f$ and they are obtained by the proposed ECG denoising technique with soft thresholding and this precisely when the $S N R i$ varied from -5 to $10 d B$. However, when the $S N R i$ is equals to $15 d B$, the $E C G$ denoising technique based on non local means [26, 27] gives the highest value of SNRf. 
Table 2. Comparative study in term of Mean Square Error $(M S E)$ : results obtained from the computations of the mean of seven values of MSE (MSE between the clean and the denoised signals). This mean is computed for seven clean ECG signals $(105,107,109,123,124,200$ and 201).dat corrupted by Gaussian white noise with different values of $S N R i$ before denoising (varying from -5 to $15 d B$ with step of $5 d B$ ).

\begin{tabular}{|c|c|c|c|c|c|}
\hline The Denoising & \multicolumn{5}{|c|}{ SNRi (dB) } \\
\cline { 2 - 6 } Technique & -5 & 0 & 5 & 10 & 15 \\
\hline $\begin{array}{c}\text { MSE obtained by } \\
\text { the proposed ECG } \\
\text { Denoising } \\
\text { technique (Hard } \\
\text { Thresholding) }\end{array}$ & 0.0126 & 0.0053 & 0.0018 & $7.7143 \mathrm{e}-04$ & $3.8571 \mathrm{e}-04$ \\
\hline $\begin{array}{c}\text { MSE obtained by } \\
\text { the proposed ECG } \\
\begin{array}{c}\text { Denoising } \\
\text { technique (Soft } \\
\text { Thresholding) }\end{array}\end{array}$ & $\mathbf{0 . 0 1 1 0}$ & $\mathbf{0 . 0 0 4 2}$ & $\mathbf{0 . 0 0 1 6}$ & $\mathbf{7 . 4 2 8 6 \mathrm { e } - 0 4}$ & $3.8571 \mathrm{e}-04$ \\
\hline $\begin{array}{c}\text { MSE obtained by } \\
\text { the TVM } \\
\text { technique [23, 24] }\end{array}$ & 0.0238 & 0.0098 & 0.0035 & 0.0012 & $4.0000 \mathrm{e}-04$ \\
\hline $\begin{array}{c}\text { MSE obtained by } \\
\text { the 1D double- } \\
\text { density complex } \\
\text { DWT denoising } \\
\text { method [25] }\end{array}$ & 0.0150 & 0.0058 & 0.0022 & $8.7143 \mathrm{e}-04$ & $3.7143 \mathrm{e}-04$ \\
\hline $\begin{array}{c}\text { MSE by the } \\
\text { denoising } \\
\text { technique based on } \\
\text { non local means } \\
\text { [26, 27] }\end{array}$ & 0.0151 & 0.0055 & 0.0021 & $8.6667 \mathrm{e}-04$ & $\mathbf{3 . 1 4 2 9 e - 0 4}$ \\
\hline
\end{tabular}

According to this table, the values in red color are the lowest values of $M S E$ and they are obtained by the proposed ECG denoising technique with soft thresholding and this precisely when the $S N R i$ varied from -5 to $10 d B$. However, when the $S N R i$ is equals to $15 d B$, the $E C G$ denoising technique based on non local means [26, 27] gives the lowest value of $M S E$.

Figures 5, 6 and 7 illustrate three examples of ECG Denoising using the proposed technique based on LWT and TVM. 

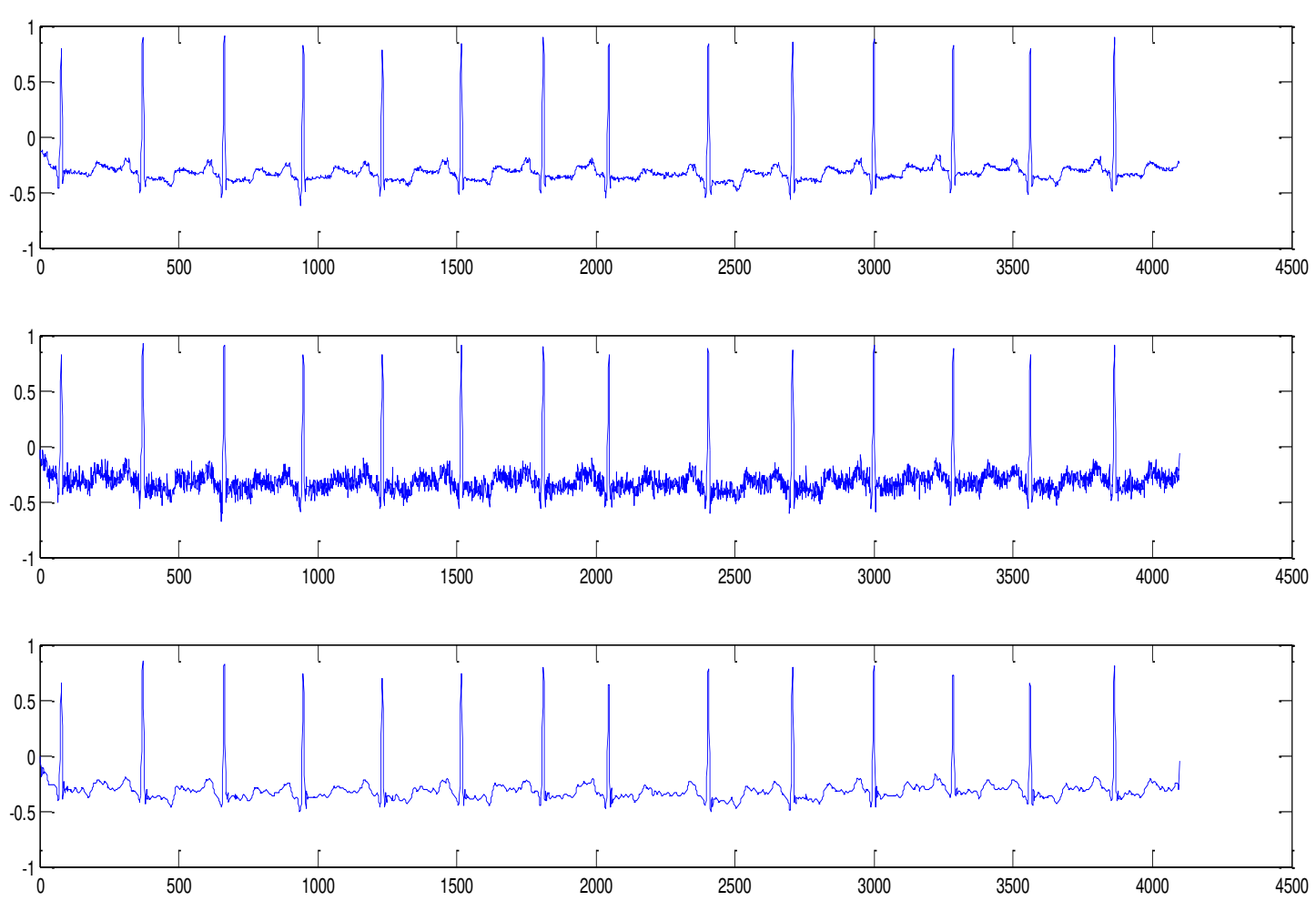

Figure 3. An example of ECG Denoising using the proposed ECG Denoising technique: (100.dat corrupted by Gaussian White Noise with $S N R=10 \mathrm{~dB}$ ).
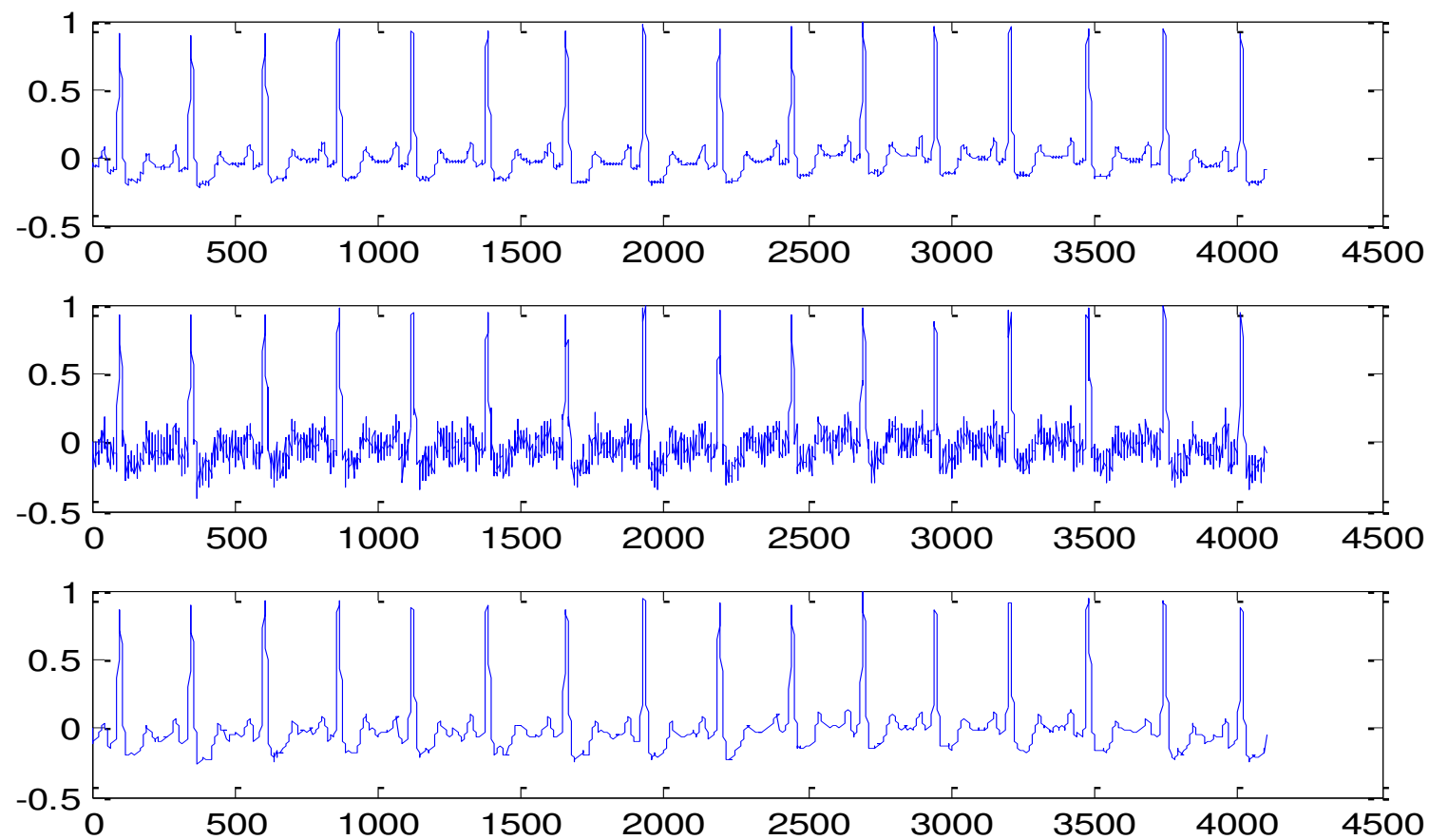

Figure 4. An example of ECG Denoising using the proposed $E C G$ Denoising technique: (105. dat corrupted by Gaussian White Noise with $S N R=10 \mathrm{~dB}$ ). 

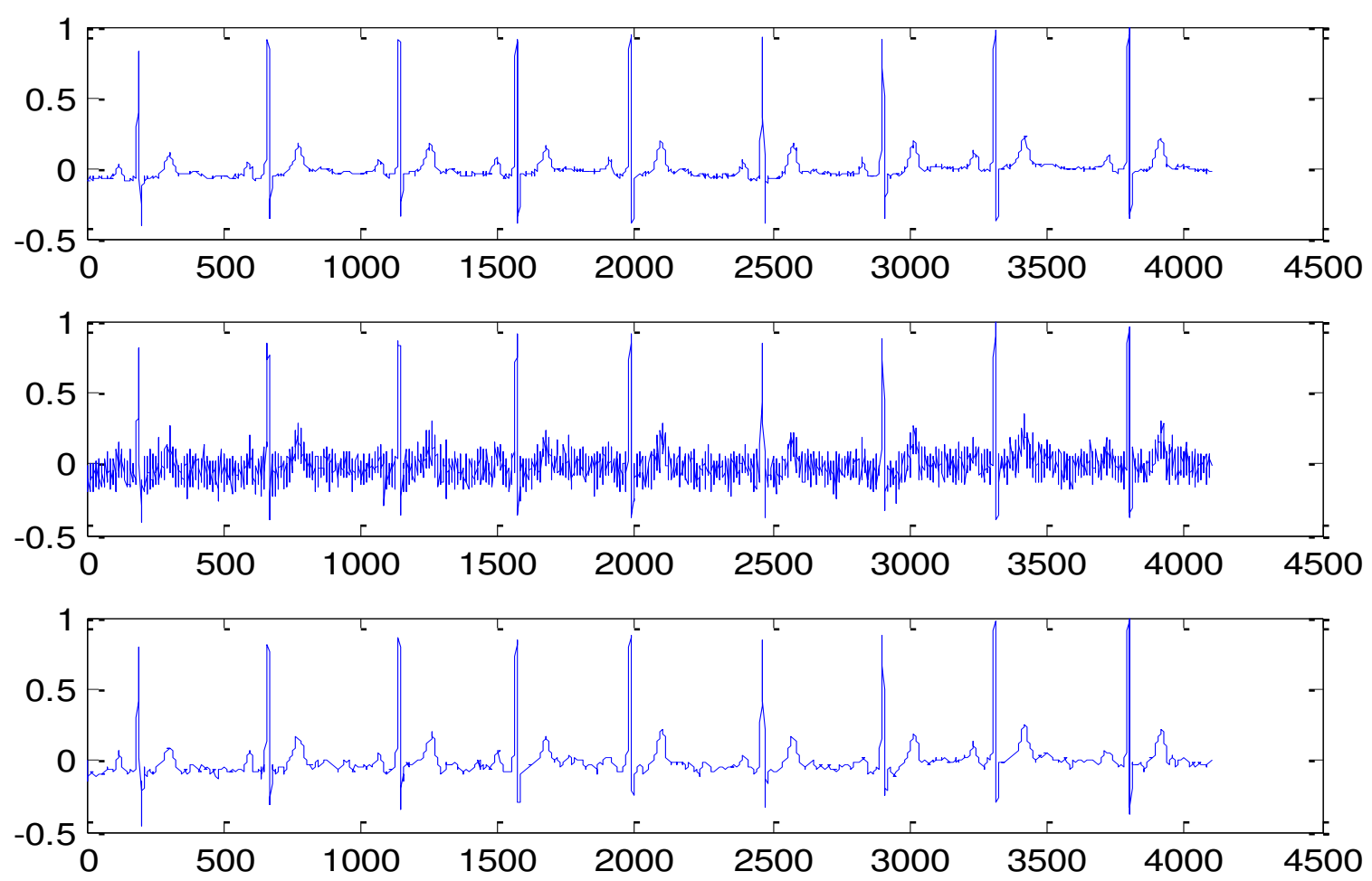

Figure 5. An example of ECG Denoising using the proposed ECG Denoising technique: (123.dat corrupted by Gaussian White Noise with $S N R=5 d B$ ).

According to those figures, one can see clearly that the proposed technique permits to cancel the noise and preserves the different waves $(P, Q, R, S, T$ and $U$ waves) of the original $E C G$ signal.

\section{Conclusion}

In this paper we propose a new technique of Electrocardiogram (ECG) Denoising based on Lifting Wavelet Transform ( $L W T$ ) and Total Variation Minimization (TVM). This technique consist at the first step in applying the LWT to the noisy ECG Signal (where 2 is the decomposition level) in order to obtain three wavelet sub-bands, $c D_{1}, c D_{2}$ and $c A_{2}$. The two coefficients, $c D_{1}, c D_{2}$ are details coefficients and they are denoised by soft or hard thresholding. The coefficient $c A_{2}$ is an approximation one and is denoised by TVM. Finally, the inverse of $L W T$ is applied to the obtained denoised coefficients, $c D d_{1}, c D d_{2}$ and $c A d_{2}$ in order to have the denoised ECG signal. The evaluation of this proposed technique is performed by comparing it to three other denoising approaches existing in literature. The first one of these approaches is based on TVM, the second one is $1-D$ double-density complex $D W T$ denoising method and the third one is based on non local means. These four denoising techniques are applied on a number of ECG signals taken from MIT - BIH database and corrupted by a White Gaussian noise at different values of Signal to Noise Ratio $(S N R(d B))$. The obtained results from the computation of the $S N R$ and the Mean Square Error $(M S E)$, show that the proposed technique outperforms the other techniques applied for our evaluation and this precisely when the $S N R i$ (before denoising) varied from -5 to $10 \mathrm{~dB}$. However, when the $S N R i=15 d B$, the denoising technique based on non local means gives the best 
results: highest value of $S N R f$ (after denoising) and lowest value of MSE (MSE between the original and the denoised signals).

\section{- Methods:}

- The proposed ECG Denoising technique with Soft/Hard thresholding,

- The TVM based denoising technique [23, 24],

- The 1D double-density complex DWT denoising method [25],

- The ECG denoising technique based on non local means [26, 27].

- Abbreviation:

- ECG: Electrocardiogram

- DWT: Discrete Wavelet Transform

- $\quad$ LWT: Lifting Wavelet Transform

- TVM: Total Variation Minimization

- $c D_{1}$ : Detail coefficient at level 1

- $\quad c D_{2}$ : Detail coefficient at level 2

- $\quad c A_{2}$ : Approximation coefficient

- $\quad c D d_{1}$ : Denoised Detail coefficient at level 1

- $c D d_{2}$ : Denoised Detail coefficient at level 2

- $\quad L W T^{-1}$ : Inverse of $L W T$

- $\quad M S E$ : Mean Square Error

- $\quad S N R$ : Signal to Noise Ratio

- SNRi: Signal to Noise Ratio before Denoising

- $\quad S N R f$ : Signal to Noise Ratio after Denoising

- EMD: Empirical Mode Decomposition

- $\quad N L M$ : Nonlocal Means

- $d B:$ Decibel

\section{References}

[1] Santosh Kumar Yadav, Rohit Sinha, Prabin Kumar Bora, arXiv:1611.03320v1 [stat.AP] 26 Oct 2016

[2] J. Bai, Y. Zhang, D. Shen, L. Wen, C. Ding, Z. Cui, F. Tian, B. Yu, B. Dai, and J. Zhang, "A portable ECG and blood pressure telemonitoring system," Engineering in Medicine and Biology Magazine, IEEE, vol. 18, no. 4, pp. 63-70, 1999. 
[3] A. Alesanco and J. Garc'ia, "Clinical assessment of wireless ECG transmission in realtime cardiac telemonitoring," IEEE Trans. Information Technology in Biomedicine, vol. 14, no. 5, pp. 1144-1152, 2010.

[4] M. Rahman, R. A. Shaik, and D. V. R. K. Reddy, "Efficient and simplified adaptive noise cancelers for ECG sensor based remote health monitoring," IEEE Sensors Journal, vol. 12, no. 3, pp. 566-573, 2012.

[5] N. E. Huang, Z. Shen, S. R. Long, M. C. Wu, H. H. Shih, Q. Zheng, N.-C. Yen, C. C. Tung, and H. H. Liu, "The empirical mode decomposition and the hilbert spectrum for nonlinear and non-stationary time series analysis," Proc. Royal Soc. London. Series A: Math., Phy. Engg. Sci., vol. 454, no. 1971, pp. 903-995, 1998. 9

[6] P. M. Agante and J. de Sa, "ECG noise filtering using wavelets with soft-thresholding methods," in Computers in Cardiology, 1999, pp. 535-538.

[7] R. Sameni, M.-B. Shamsollahi, C. Jutten, and G. Clifford, "A nonlinear bayesian filtering framework for ECG denoising," IEEE Trans. Biomed. Engg., vol. 54, no. 12, pp. 21722185, 2007.

[8] O. Sayadi and M.-B. Shamsollahi, "ECG denoising and compression using a modified extended kalman filter structure," IEEE Trans. Biomed. Engg., vol. 55, no. 9, pp. 22402248, 2008.

[9] M. Blanco-Velasco, B. Weng, and K. E. Barner, "ECG signal denoising and baseline wander correction based on the empirical mode decomposition," Computers in Biology and Medicine, vol. 38, no. 1, pp. 1-13, 2008.

[10] J. Gao, H. Sultan, J. Hu, and W. wen Tung, "Denoising nonlinear time series by adaptive filtering and wavelet shrinkage: A comparison," IEEE Signal Processing Letters, vol. 17, no. 3, pp. 237-240, 2010.

[11] H. Kestler, M. Haschka, W. Kratz, F. Schwenker, G. Palm, V. Hombach, and M. Hoher, "Denoising of high-resolution ECG signals by combining the discrete wavelet transform with the wiener filter," in Computers in Cardiology, 1998, pp. 233-236.

[12] B. Weng, M. Blanco-Velasco, and K. Barner, "ECG denoising based on the empirical mode decomposition," in IEEE Int. Conf. of Engg. in Med. and Bio. Soc., Aug 2006, pp. $1-4$.

[13] M. A. Kabir and C. Shahnaz, "Denoising of ECG signals based on noise reduction algorithms in EMD and wavelet domains," Biomed. Signal Process. Control, vol. 7, no. 5, pp. 481-489, 2012.

[14] A. Buades, B. Coll, and J. M. Morel, "A non-local algorithm for image denoising," in IEEE Conference on Computer Vision and Pattern Recognition, vol. 2, 2005.

[15] B. Tracey and E. Miller, "Nonlocal means denoising of ECG signals," IEEE Trans. Biomed. Engg., vol. 59, no. 9, pp. 2383-2386, 2012.

[16] K. Dabov, A. Foi, V. Katkovnik, and K. Egiazarian, "Image denoising by sparse 3D transform-domain collaborative filtering," IEEE Trans. Image Process., vol. 16, no. 8, pp. 2080-2095, 2007.

[17] P. Chatterjee and P. Milanfar, "Clustering-based denoising with locally learned dictionaries,” IEEE Trans. Image Process., vol. 18, no. 7, pp. 1438-1451, 2009.

[18] A. Rajwade, A. Rangarajan, and A. Banerjee, "Image denoising using the higher order singular value decomposition," IEEE Trans. Pattern Anal. Mach. Intell., vol. 35, no. 4, pp. 849-862, 2013.

[19] D. Van De Ville and M. Kocher, "Sure-based non-local means," IEEE Signal Processing Letters, vol. 16, no. 11, pp. 973-976, 2009.

[20] Mourad Talbi, Med Salim Bouhlel, "Singular Values Decomposition and Lifting Wavelet Transform for Speech Signal Embedding into Digital Image,' Recent Advances in Electrical \& Electronic Engineering, Volume 12, Issue 2, 2019. 
[21] Talbi Mourad, Med Salim Bouhlel, "Secure Image Watermarking Based on LWT and SVD, Int. J. Image Graphics 18(4): 1850021(2018).

[22] Sira Ben Fatima, Mourad Talbi, Tahar Ezzedine, "LWT-SVD secure image watermarking technique,' 2017 International conference of Electronics, Communication and Aerospace Technology (ICECA), India.

[23] A. Chambolle: An Algorithm for Total Variation Minimization and Applications. Journal of Mathematical Imaging and Vision 20 (1-2): 89-97, January - March, 2004

[24] https://github.com/gpeyre/2016-IP-tv-denoising/blob/master/code/perform tv denoising.m

[25] http://eeweb.poly.edu/iselesni/DoubleSoftware/signal.html

[26] Ambuj D. and Hasnine M.. Two-stage Nonlocal Means Denoising of ECG Signals. Int. J. of Advan. Rese.. in Comput. Sci. 2014, 5, 114-118.

[27] Brian H. T. and Eric L. M. Nonlocal Means Denoising of ECG Signals. IEEE Trans on Biomed. Eng., 2012, 59(9), 2383-2386.

- Ethics approval and consent to participate: All the authors of this paper are agree for its submission to your journal

- Consent for publication: all the authors are agree for publication of this paper

- Competing interests: no competing interests

- Funding: No Funding

- Authors' contributions:

- First Author: his contributions are the suggestion of the new proposed ECG Denoising technique based on LWT and TVM and writing of the paper,

- Second Author: Checking the English of the paper and the different mathematical equations in this paper

- Acknowledgements:

We would like to thank all the people who contributed in some way to this work which was supported by CRTEn (Center of Researches and Technologies of Energy of Borj Cedria, Tunis) and the Ministry of Higher Education and Scientific Research).

- Availability of data and material:

- The different Matlab codes for the simulation of the proposed ECG denoising technique based on LWT and TVM are taken from MATLAB/Simulink and also the following site:

http://eeweb.poly.edu/iselesni/lecture_notes/TVDmm/ 
- The simulation of the 1D double-density complex DWT denoising method [25] is performed using the following site

http://eeweb.poly.edu/iselesni/DoubleSoftware/signal.html

- the simulation of the ECG denoising technique based on non local means $[26,27]$ is performed using matworks:

https://www.mathworks.com/matlabcentral/fileexchange/41762-non-local-means-nlmdenoising-for-time-series-applied-to-ecg

- For the simulation and evaluation of the proposed ECG Denoising technique and the other ones we have used the MIT-BIH Database. 
Figures

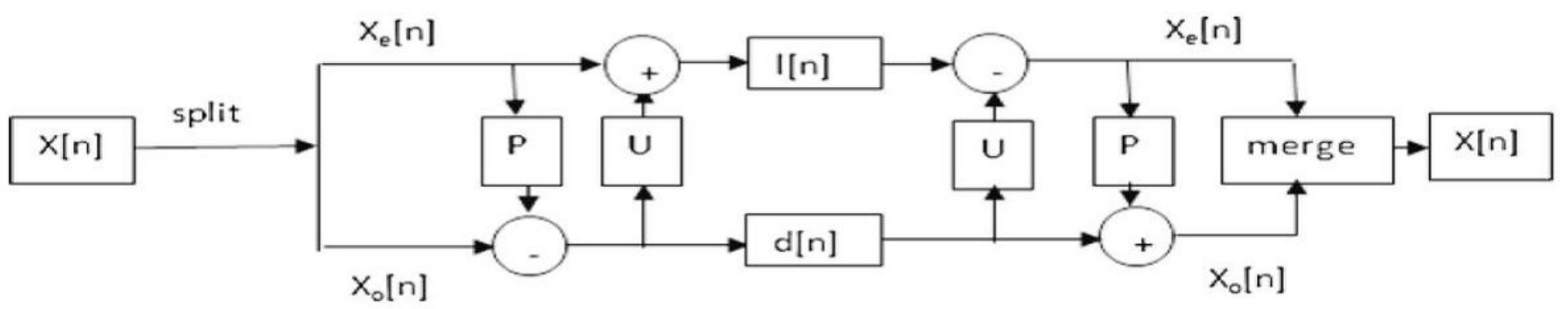

Decomposition

Reconstruction

Figure 1

Decomposition and Reconstruction of a signal X[n] using LWT and its inverse respectively

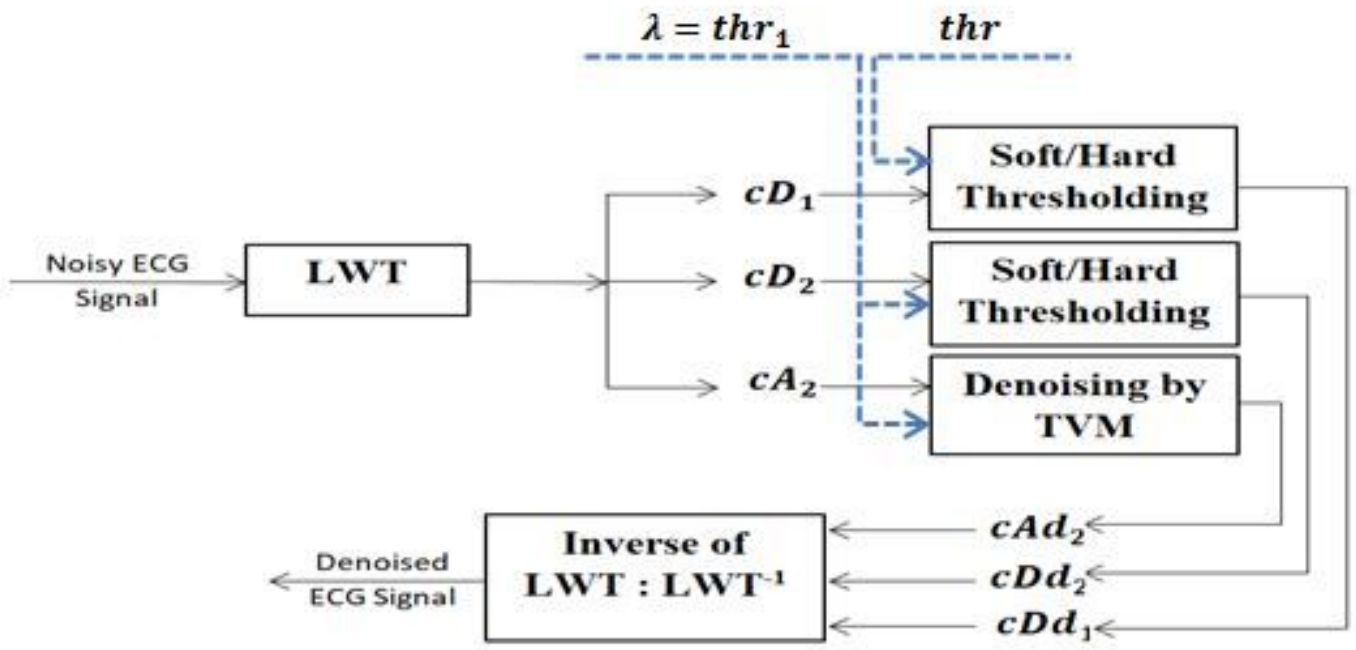

Figure 2

The flowchart of the proposed ECG Denoising Technique

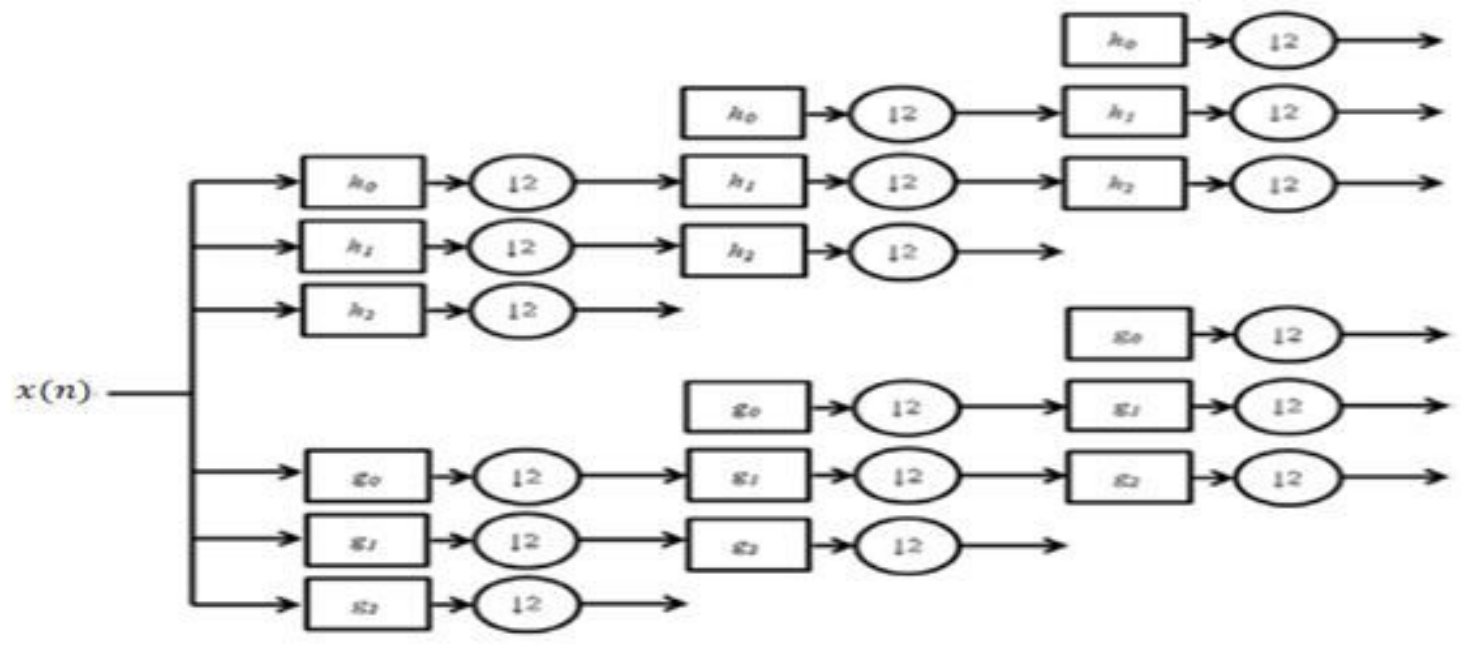


Figure 3

Filter bank diagram of 1-D double density complex DWT

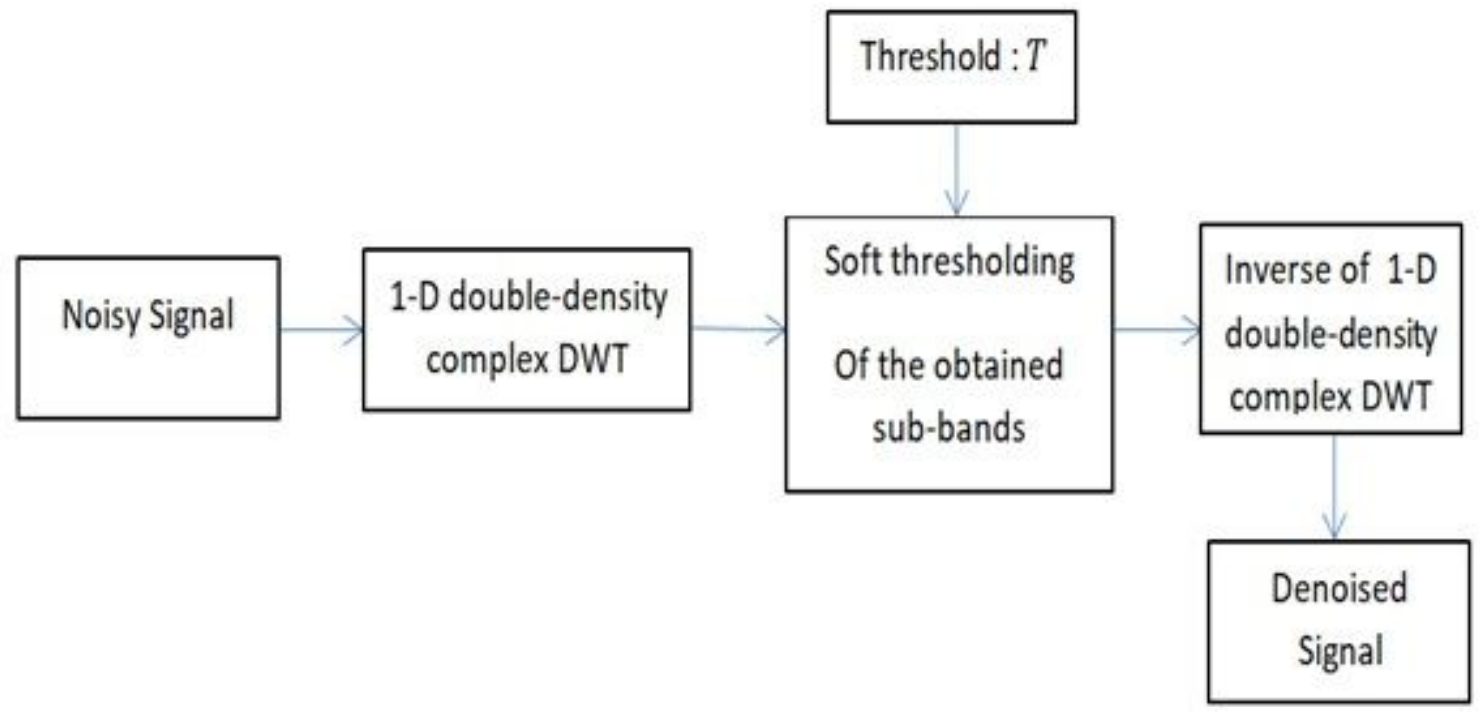

Figure 4

Signal denoising using 1-D double-density complex DWT denoising technique
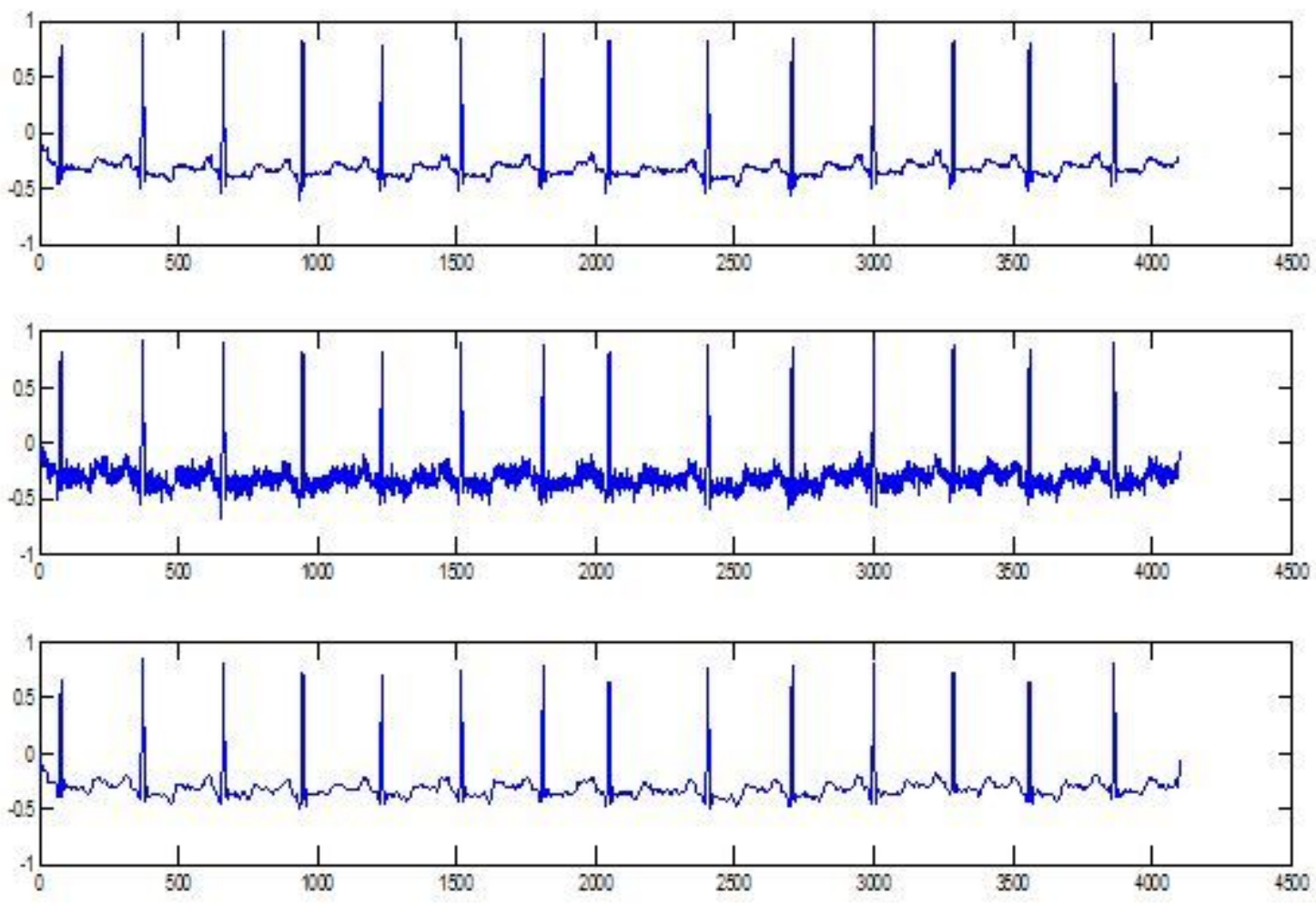

Figure 5

An example of ECG Denoising using the proposed ECG Denoising technique: (100.dat corrupted by Gaussian White Noise with SNR=10dB). 

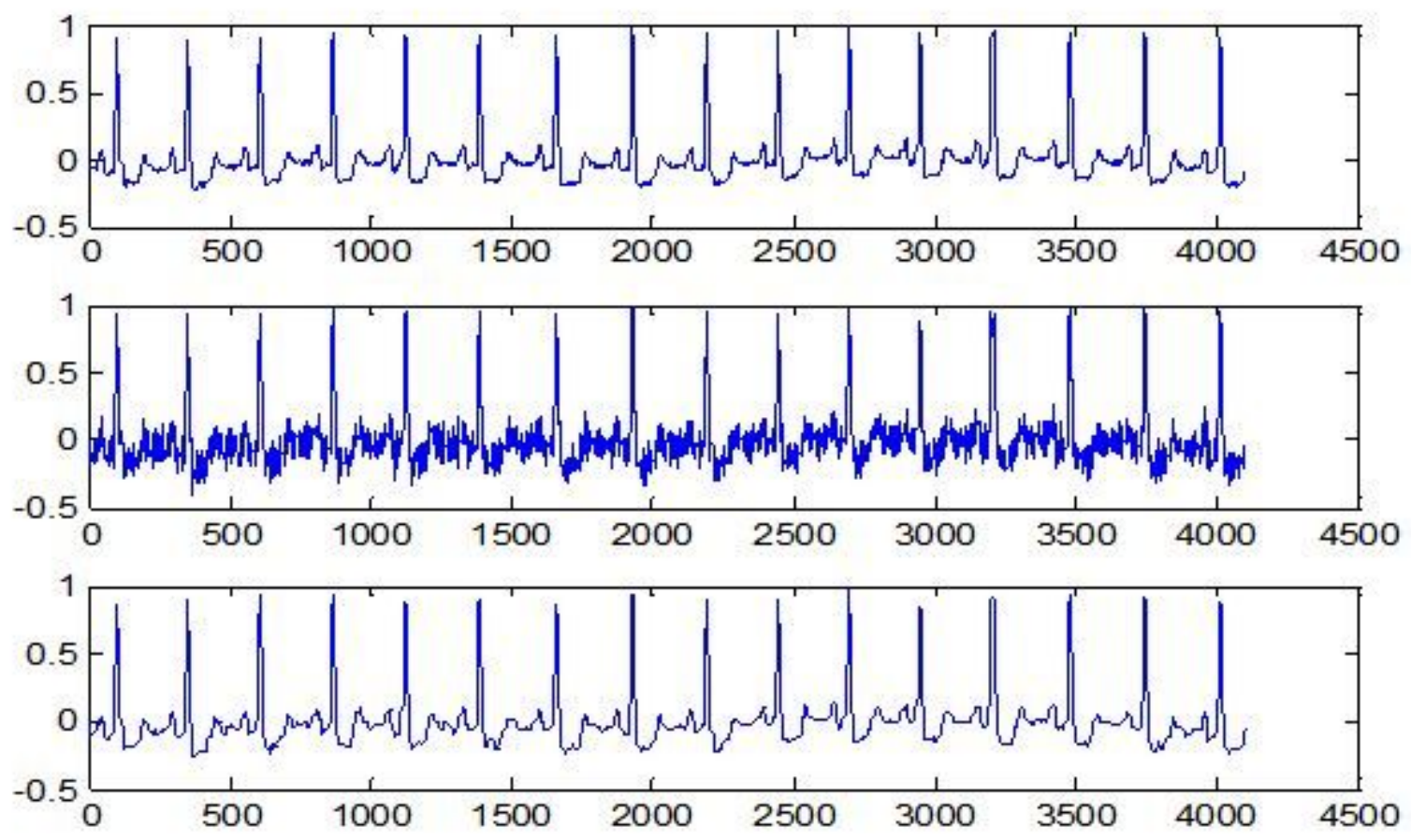

Figure 6

An example of ECG Denoising using the proposed ECG Denoising technique: (105.dat corrupted by Gaussian White Noise with SNR=10dB).
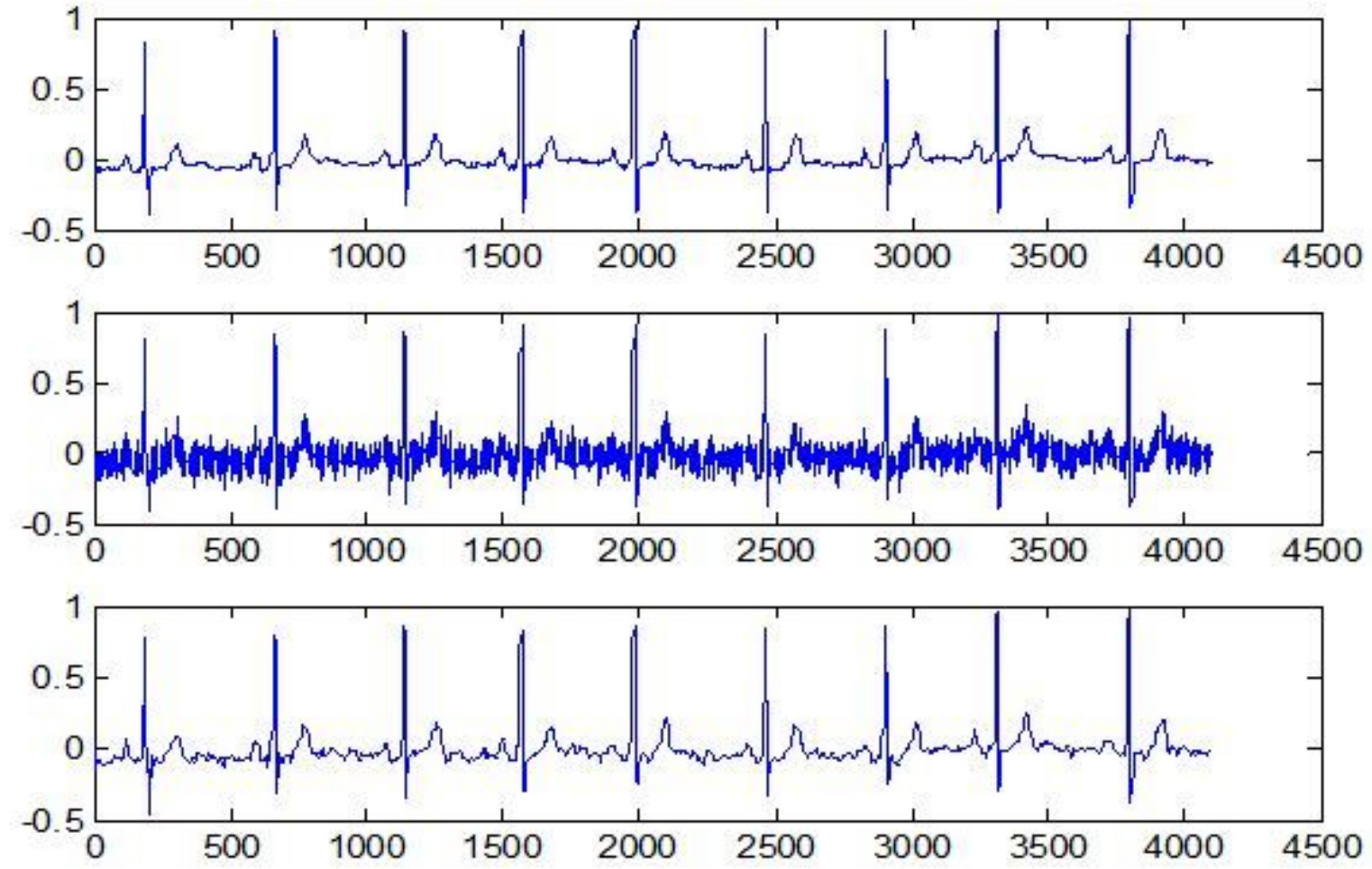

Figure 7 
An example of ECG Denoising using the proposed ECG Denoising technique: (123.dat corrupted by Gaussian White Noise with SNR=5dB). 